\title{
User-friendly design method for fuzzy logic controller
}

\author{
C.-L. Chen \\ C.-T.Hsieh
}

Indexing terms: Fuzzy logic control, Fuzzy sets, Shrinking-span membership functions

\begin{abstract}
A new means for designating membership functions in a fuzzy logic controller (FLC) is presented. The method allows a novice to construct a set of membership functions, called shrinking-span membership functions (SSMFs), for a specific linguistic variable systematically by using only two parameters: number of elements of the term set and the shrinking factor for that linguistic variable. The SSMFs have different spans for various term set elements in the universe of discourse and this gives the FLC more power to deal with the nonlinearity of the control problems encountered in the real applications. When there is not enough domain knowledge about the process, the SSMFs make it possible for a designer to set up a reasonable and practical rule base for the FLC. According to the computational simulations presented, the satisfactory performance of such an FLC for several test problems can be acquired without laborious optimisation of the tuning parameters. Therefore the proposed approach narrows the gap between a theoretical FLC and a practical one and makes the FLC more down-to-earth.
\end{abstract}

\section{Introduction}

People very often make decisions in their daily lives based on qualitative information. Zadeh's fuzzy sets [1] theory was thus proposed to enable people to describe and formulate the linguistic mental models apparent in daily life behaviour.

Mamdani and colleagues $[2,3]$ were pioneers in applying fuzzy techniques to process control. Their results, as well as those of many other researchers, have demonstrated the potential value of the fuzzy logic control system on simple process dynamics. A comprehensive review of the classical design and implementation of the fuzzy logic controller (FLC) can be found in [4]. More advanced design techniques have also been reported in literature, such as the adaptive hierarchical fuzzy controller [5] and the fuzzy logic controller with multiple inputs [6].

The superior performance of FLC as reported in lit-

(C) IEE, 1996

IEE Proceedings online no. 19960393

Paper first received 16th October 1995 and in revised form 6th February 1996

The authors are with the Department of Chemical Engineering, National Taiwan University, Taipei 10617, Taiwan, Republic of China erature is usually conjectured to have its origin in their switching nature [7], where the magnitude of the rate of change in controller output is greater for larger error but smaller for process output close to set point. The relations between fuzzy and conventional three-mode (proportional-integral-derivative, PID) controllers have been studied in $[8,9]$ and other works.

To simplify the analysis of the FLC, most researchers prefer to use simple triangular functions, especially the equal-span isosceles, as the membership functions of the FLC. However, the membership functions of the FLC in most of the real applications are constructed by using knowledge of domain experts, and for the purpose of obtaining a good control result, these membership functions often come out with various physical shapes. Intuitively, when the control response is closer to the set point, the corresponding membership function for the specific linguistic variable should have a narrower range. Chen [10] has confirmed this by applying neural-network training techniques to an FLC.

In this article, we propose a systematic approach to construct membership functions for the FLC. By using this method, the designer of an FLC assigns only the number of elements of the term set and the shrinking factor for a specific linguistic variable and then obtains a series of well-located membership functions.

Such shrinking-span membership functions (SSMFs) not only make themselves more reasonable from the human experts' point of view but also provide the FLC the ability to easily adapt to different control systems by slight modification. After incorporating SSMFs into the FLC structure, the FLC, designated as SSMFsFLC, can therefore generate large and fast control actions when the system output is far from the set point and only make moderate and slow changes when the system output is near the set point. Such behaviour saves a lot of time in optimising the scaling factors in the design procedures, and this reduces the difficulties in implementing an FLC and makes it more robust in real operational environments.

\section{Basic design procedures of FLC}

Complete and thorough descriptions of the design procedure for an FLC have been presented $[11,12]$ therefore only the essential materials and notations are stated here as a fundamental work.

\subsection{Rule base}

The rule base is formed by a family of linguistic rules that describes the relationship between the input and output variables of the controller. In the case of $I$ inputs single-output fuzzy systems, for example, the $j$ th control rule in the rule base with $J$ rules can be 
expressed as

$$
\begin{array}{r}
R j: \text { IF } x_{1} \text { is } A_{1 j} \text { AND } x_{2} \text { is } A_{2 j} \text { AND } \cdots \\
\text { AND } x_{I} \text { is } A_{I j} \text { THEN } y \text { is } B_{j}
\end{array}
$$

where $x_{i}(i=1 \sim I)$ is the process state linguistic variable defined on the universe of discourse $U x_{i}$ with term set $T x_{i}, A i_{j}(j=1 \sim J)$ is the linguistic value of $x_{i}$ with $A i_{j} \in T x_{i} . y, U_{y}, T_{y}$ and $B_{j}$ are the counterparts of $x_{i}$, $U x_{i}, T x_{i}$ and $A i_{j}$ for control action. We name the representation method described in eqn. 1 the sequence representation of the rule base.

\subsection{Decision making logic}

The individual-rule based inference ([11] p. 94) is employed and modified here to cope with the inference of fuzzy sets. In this approach, a four-steps operation is proposed to accomplish the inference procedure ([12] Section 4.3): .

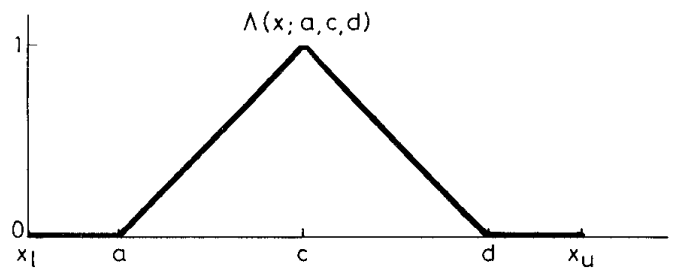

Fig. 1 Membership functions of trapezoidal farnily

Step 1: Computing the level of matching $A i_{j}\left(x_{i}^{*}\right)$ between the crisp input value $x_{i}^{*}$ and linguistic value $A i_{j}$ :

$$
A_{i j}\left(x_{i}^{*}\right)=\mathbf{M}\left(x_{i}^{*}, A_{i j}\right)
$$

M maps a crisp input value to a membership degree subjected to a specific membership function. Following [13] (let $x_{l}=\inf U_{x}, x_{u}=\sup U_{x}$ ), function $\Lambda$ is used here to construct the membership functions used in this research (see Fig. 1)

$$
\Lambda(x ; a, c, d)=\Pi(x ; a, c, c, d)= \begin{cases}0 & \text { for } x \leq x<a \\ \frac{x-a}{c-a} & \text { for } a \leq x \leq c \\ \frac{d-x}{d-c} & \text { for } c \leq x \leq d \\ 0 & \text { for } d \leq x \leq x_{u}\end{cases}
$$

Step 2: Finding the firing level $\phi_{j}$ of each of the rules in the rule base:

$$
\phi_{j}=\mathbf{F}\left(A_{1 j}\left(x_{1}^{*}\right), \ldots, A_{I j}\left(x_{I}^{*}\right)\right)=\underset{i=1}{I}\left(A_{i j}\left(x_{i}^{*}\right)\right)
$$

In the Mamdani-FLC, $\mathbf{F}$ is the MIN aggregating operator. However, it can be replaced by other triangular norms (t-norms for short) [14].

Step 3: Deciding the output fuzzy set $\mathrm{B}_{j}^{\prime}$ of each rules. The formulation that determines how $\phi_{j}$ and the fuzzy set $B_{j}$ interact to form the rule output is called a fuzzy implication. Here, I denotes this operation.

$$
B_{j}^{\prime}(y)=\mathbf{I}\left(\phi_{j}, B_{j}(y)\right)
$$

I is usually implemented as the MIN or the product though other t-norms are possible.

Step 4: Aggregating the individual output fuzzy sets $B_{j}^{\prime}$ s to form the overall system output fuzzy set $B$. Operator A, usually implemented as the MAX or the summation, is used here:

$$
B(y)=\mathbf{A}\left(B_{\mathbb{1}}^{\prime}(y), \ldots, B_{J}^{\prime}(y)\right)=\underset{j=1}{J}\left(B_{j}^{\prime}(y)\right)
$$

\subsection{Defuzzification interface}

The defuzzification operator $\mathbf{D}$ converts the fuzzy set $B$ into a single crisp value $y^{*}$, i.e.

$$
y^{*}=\mathbf{D}(B(y))
$$

Centre of area/gravity (COA or COG for short) method is the most popular scheme used to calculate the crisp value [11]:

$$
y_{C O A}^{*}=\frac{\int_{U_{y}} B(y) y d y}{\int_{U_{y}} B(y) d y}
$$

In short, the crisp control action $y^{*}$ of an FLC can be obtained by a series of operations:

$y^{*}=\mathbf{D}\left(\underset{j=1}{\mathbf{A}}\left(\mathbf{I}\left(\underset{i=1}{I}\left(\mathbf{M}\left(x_{i}^{*}, A_{i j}\right)\right), B_{j}(y)\right)\right)\right)$

\section{Design of FLC with shrinking-span membership functions}

From the description of previous Section, one would find that a lot of design parameters must be chosen before an FLC can be constructed. The most cumbersome tasks in designing these parameters lay in the designation of proper membership functions and the formulation of a comprehensive and reasonable rule base. Therefore, how much and how effectively one can lessen the difficulties in building the membership functions and the rule base becomes a main concern in the whole FLC design procedure.

In this Section, we propose a systematic design method for the FLC. This organised method not only simplifies the sequences in constructing an FLC but also makes the FLC more flexible in practical applications.

First, we make a few modifications on the rule-base representation and then introduce the shrinking-span membership functions, and finally derive the simply input-output relation of the FLC.

\subsection{Simple rule base}

With the purpose of making the FLC expression less complex, we use a numerical sequence to map the elements in the term-set of the linguistic variable $x$. For an $I$-input single-output FLC, the term-set for input variable $x_{i}$ is denoted by

$$
\begin{aligned}
T_{x_{i}} & =\left\{A_{\left(i,-m_{i}\right)}, \ldots, A_{(i,-1)}, A_{(i, 0)}, A_{(i, 1)}, \ldots, A_{\left(i, m_{i}\right)}\right\} \\
& =\left\{A_{\left(i, l_{i}\right)} l_{i} \in I_{m_{i}}\right\}
\end{aligned}
$$

here $I m_{i}=\left\{-m_{i}, \ldots,-1,0,1, \ldots, m_{i}\right\}$ is the index set with $M_{i}\left(M_{i}=2 m_{i}+1\right)$ terms for linguistic variable $x_{i}$. That is, the universe of discourse $U x_{i}$ of linguistic variable $x_{i}$ is partitioned into $M_{i}$ sections and each section is issociated with a linguistic term and is characterised by the fuzzy set $A\left(i, l_{i}\right)$. Similarly, the output variable $y$ for the FLC has the term-set as follows:

$T_{y}=\left\{B_{-m_{y}}, \ldots, B_{-1}, B_{0}, B_{1}, \ldots, B_{m_{y}}\right\}=\left\{B_{l} \mid l \in I_{m_{y}}\right\}$

$m_{y}, I_{m y}, l$ and $M_{y}$ are analogous to $m_{i}, I m_{i}, l_{i}$ and $M_{i}$, respectively. In this way, the rule base for the I-input single-output FLC can be expressed by

$$
\begin{aligned}
\mathbf{R}\left(l_{1}, \ldots, l_{I} ; l\right): & \mathbf{I F} x_{1} \text { is } A_{\left(1, l_{1}\right)} \text { AND } \\
& \ldots \text { AND } x_{I} \text { is } A_{\left(I, l_{I}\right)} \text { THEN } y \text { is } B_{l} \\
& \forall l_{i} \in I_{m_{i}}, \quad l \in I_{m_{y}}
\end{aligned}
$$


This representation method of rule base is called the index-represented rule mapping [6]. We call it index representation in the following text to distinguish it from the sequence representation depicted in eqn. 1 . Assume that the complete and simple rule mapping properties [6] hold for the remaining parts of this research, that is

$$
l=\sum_{i=1}^{l} l_{i}
$$

From the previous relation, it can be shown that for a simple rule mapping $m_{y}$ is the summation of all $m_{i} \mathrm{~s}$, that is $\mathrm{m}_{y}=\sum_{i=1}^{I} m_{i}$, and the number of linguistic values for output $y, M_{y}$, in an $I$-input single-output FLC is determined by $M_{y}=2 m_{y}+1=2 \sum_{i=1}^{I} m_{i}+1$ and the total number of rules in the rule base is $J=\prod_{i=1}^{I} M_{i}$ $\left(M_{i}=2 m_{i}+1\right)$.

To take advantage of both the index representation and sequence representation, the following relations are proposed as transformations between the indices $l_{i} \mathrm{~s}$ of the index representation and the index $j$ of the sequence representation:

$$
\begin{aligned}
j= & h\left(l_{1}, \ldots, l_{I}\right) \\
= & \left(l_{1}+m_{1}\right) \times M_{2} \times M_{3} \times \ldots \times M_{I} \\
& +\left(l_{2}+m_{2}\right) \times M_{3} \times \ldots \times M_{I} \\
& +\ldots \\
& +\left(l_{I-1}+m_{I-1}\right) \times M_{I} \\
& +\left(l_{I}+m_{I}\right)+1 \\
= & \sum_{i=1}^{I}\left[\left(l_{i}+m_{i}\right)\left(\prod_{k=i+1}^{I} M_{k}\right)\right]+1 \\
l_{i}=\bar{h}(j)= & {\left[\frac{j-1-\sum_{q=1}^{i-1}\left[\left(l_{q}+m_{q}\right)\left(\prod_{p=q+1}^{I} M_{p}\right)\right]}{\prod_{k=i+1}^{I} M_{k}}\right]-m_{i} } \\
& {\left[\begin{array}{l}
\text { for } i=1 \sim I
\end{array}\right.}
\end{aligned}
$$

Of course, other forms of $h$ and $\bar{h}$ are acceptable as long as they are adequate to perform accurate mapping between these two types of representations. From the relations proposed, it is obvious that whenever a set of $l_{i} \mathrm{~s}$ is chosen a corresponding $j$ is determined, and vice versa.

Table 1 illustrates both the index representation and sequence representation of a simple rule mapping.

Table 1: Simple rule mapping for $I-2$ and $m_{1}=m_{2}=2$

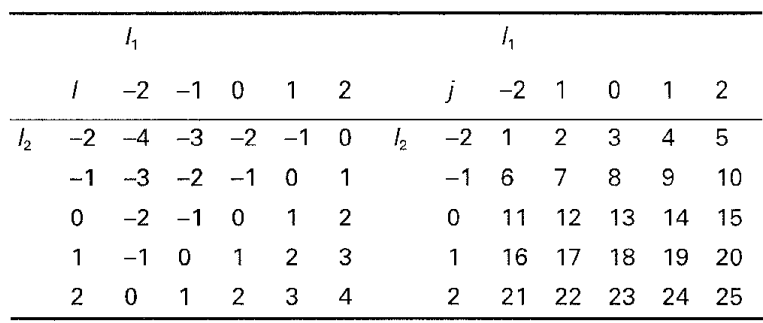

(a) Index representation

(b) Sequence representation

\subsection{Shrinking-span membership functions}

In the real applications of FLC, the membership functions are constructed by assembling knowledge of the domain experts and then modified by laboriously surveying the control response of the process. In most control cases, the FLC cannot be effective without carefully arranging the membership functions.

In the theoretical analysis of the FLC, however, the selection of membership functions does not get much attention by the majority of researchers. Most choose isosceles triangular functions with equal spans throughout the whole universe of discourse as membership functions for their FLCs $[2,6,8,9]$. The main advantage of choosing this type of membership function is that equal-span isosceles triangular membership functions ease the difficulties in analysing the structure of the FLC. However, almost all the applications of FLC adopt nonequal-span membership functions to cope with the real control problems. Instinctively, the closer the control response to the set point (or normal condition), the narrower the membership function range should be. For some highly nonlinear processes, such as inverted pendulum system, an FLC with equal span triangular membership function is not adequate to achieve a good control result.

To accomplish a better performance and to devise a more rational FLC, the shrinking-span membership functions is propounded to fulfill these aims. As an illustrative study, the following assumptions are made:

[A1] Trapezoidal family membership functions are presupposed here as a fundamental work. This implies that all membership functions are normal. Other types of membership functions, such as gaussian family membership functions, can be applied directly.

[A2] The universes of discourse for all linguistic variables $x_{i}$ s and $y$ are confined to the range $[-1,1]$. Such a restriction can be easily satisfied by multiplying a scaling factor to the original values of these linguistic variables.

Conceptually, the SSMFs are a series of orderly arranged membership functions for a linguistic variable across its universe of discourse. By using a series of $A\left(x_{i}\right)$ s, we propose the following representation for the trapezoidal family SSMFs $A^{\beta}\left(i, l_{i}\right)\left(x_{i}\right)$ for linguistic variable $x_{i}$ :

$$
\begin{aligned}
& A_{\left(i, l_{i}\right)}^{\beta}= \\
& \Lambda\left(x_{i} ; \frac{(1+\beta) A_{\left(i, l_{i}-1\right)}^{*}+(1-\beta) A_{\left(i, l_{i}\right)}^{*}}{2}, A_{\left(i, l_{i}\right)}, \frac{(1-\beta) A_{\left(i, l_{i}\right)}^{*}+(1+\beta) A_{\left(i, l_{i}+1\right)}^{*}}{2}\right) \\
& \quad \text { for } l_{i} \in I_{m_{i}}
\end{aligned}
$$

here $A^{*}\left(i, l_{i}\right)$ is the principal value of $A\left(i, l_{i}\right)\left(\mathrm{x}_{i}\right)$ defined by

$$
A_{\left(i, l_{i}\right)}^{*}=\frac{l_{i}}{m_{i}} s_{i}^{m_{i}-\left|l_{i}\right|}, \quad l_{i} \in I_{m_{i}}
$$

where $s_{i} \in[0,1]$ is the shrinking factor for linguistic variable $x_{i}$. To comply with this representation, let $A^{*}\left(i,-m_{i}-1\right)=A^{*}\left(i,-m_{i}\right)$ and $A^{*}\left(i, m_{i}+1\right)=A^{*}\left(i, m_{i}\right) ; A^{\beta}\left(i,-m_{i}\right)\left(A^{*}\left(i,-m_{i}\right)\right)$ $=1$ and $A^{\beta}\left(i, m_{i}\right)\left(A^{*}\left(i, m_{i}\right)\right)=1$. By applying various shrinking factors to the same linguistic variable, different membership functions can be obtained to examine which is the most suitable for a specific application process. $\beta$ is the overlapping factor whose reasonable range is $[0,1]$. $\beta$ can take values greater than unity as long as the resultant membership functions are rational in applications. The overlapping region increases monotonically as $\beta$ increases. For $\beta=0$ it is clear that there is no overlap between the SSMFs and if $\beta=1$ the supports for the SSMFs have proper overlapping regions. 
A typical SSMF is shown in Fig. 2 (the notations have been simplified to make them easier to be comprehended).

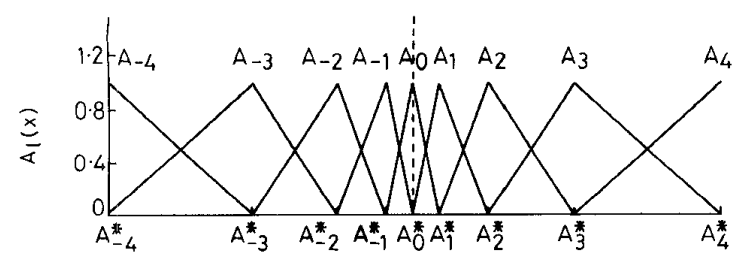

Fig.2 Typical trapezoidal family SSMFs $m=4 ; s=0.7 ; \beta=1$

Note that when shrinking factor $s_{i}=1$, the SSMFs $A\left(i, l_{i}\right)\left(x_{i}\right)$ s degrade to ordinary equal-span membership functions. Therefore the conventional equal-span membership functions are a special case of the SSMFs.

The specifications of the SSMFs described above have the advantage of reducing the design parameters of an FLC. With the definitions of SSMFs, the number of linguistic values $m_{i}$ and the shrinking factor $s_{i}$ are the only required parameters for setting up the membership functions of a linguistic variable. This not only dramatically lessens the efforts in obtaining the membership functions but aso provides a more reasonable interpretation of these membership functions.

\subsection{Design of FLC with SSMFs}

In this Section, we construct an FLC based on the conception of simple rule mapping and SSMFs. We name this type of fuzzy controller a fuzzy controller with shrinking-span membership functions (SSMFs-FLC for short). Further, we make some simplification of SSMFs-FLC by choosing proper operators to make the SSMFs-FLC more practical.

Basically, an SSMFs-FLC is an FLC having input/ output relation described in eqn. 9 except that the rule base adopts the simple rule mapping principle and the membership functions of the input variables $x_{i} \mathrm{~s}$ and output variable $y$ are restricted to SSMFs.

To make the structure more easily understood, the following assumptions are observed. First, all input linguistic variables are assumed to have the same number of linguistic values, that is $m_{1}=m_{2}=\ldots=m_{I}=m$. With the simple rule mapping $m_{y}=\Sigma_{i=1}^{I} m_{i}=I m$ holds and therefore the number of linguistic values for output is $M_{y}=2 m_{y}+1=2 I m+1$. Furthermore, the trapezoidal family membership functions having the form of eqn. 16 are chosen for both the input and output linguistic variables with $\beta=1$. Other values for $\beta$ are acceptable provided that the membership functions are reasonable for applications.

Explicitly, the membership functions of input linguistic variable $x_{i}$ can be expressed as (let $A^{*}\left(i,-m_{i}-1\right)=A^{*}\left(i,-m_{i}\right)$ and $A^{*}\left(i, m_{i}+1\right)=A^{*}\left(i, m_{i}\right) ; A\left(i,-m_{i}\right)\left(A^{*}\left(i,-m_{i}\right)\right)=1$ and $A\left(i, m_{i}\right)\left(A^{*}\left(i, m_{i}\right)\right)=1$ in the following equations)

$$
\begin{aligned}
A_{\left(i, l_{i}\right)}\left(x_{i}\right) & =\left.A_{\left(i, l_{i}\right)}^{\beta}\left(x_{i}\right)\right|_{\beta=1} \\
& = \begin{cases}\frac{x_{i}-A_{\left(i, l_{i}-1\right)}^{*}}{A_{\left(i, l_{i}\right)}^{*}-A_{\left(i, l_{i}-1\right)}^{*}} & \text { for } A_{\left(i, l_{i}-1\right)}^{*} \leq x_{i} \leq A_{\left(i, l_{i}\right)}^{*} \\
\frac{A_{\left(i, l_{i}+1\right)}^{*}-x_{i}}{A_{\left(i, l_{i}+1\right)}^{*}-A_{\left(i, l_{i}\right)}^{*}} & \text { for } A_{\left(i, l_{i}\right)}^{*} \leq x_{i} \leq A_{\left(i, l_{i}+1\right)}^{*} \\
0 & \text { otherwise }\end{cases} \\
& =\Lambda\left(x_{i} ; A_{\left(i, l_{i}-1\right)}^{*}, A_{\left(i, l_{i}\right)}^{*}, A_{\left(i, l_{i}+1\right)}^{*}\right)
\end{aligned}
$$

As for the output linguistic variable $y$, similar representations can be obtained:

$$
B_{l}(y)=\Lambda\left(y ; B_{l-1}^{*}, B_{l}^{*}, B_{l+1}^{*}\right)
$$

here, $B_{l}^{*}$ is principal value defined by

$$
B_{l}^{*}=\frac{l}{m_{y}} s_{y}^{m_{y}-|l|}, \quad l \in I_{m_{y}} \equiv\left\{0, \pm 1, \ldots, \pm I_{m}\right\}
$$

The following three properties can be derived for the SSMFs with $\beta=1$.

Property 1: Given numerical value $x_{i}^{*} \in ;\left|A^{*}\left(i, l_{i}\right), A^{*}\left(i, l_{i}+1\right)\right|$ $\subset U x_{i}$ for linguistic variable $x_{i}$, there exists only two nonzero membership values in the SSMFs, that is

$$
\begin{aligned}
& A_{\left(i, k_{i}\right)}\left(x_{i}^{*} \in\left[A_{\left(i, l_{i}\right)}^{*}, A_{\left(i, l_{i}+1\right)}^{*}\right]\right) \begin{cases}\neq 0 & \text { if } k_{i}=l_{i}, l_{i}+1 \\
=0 & \text { otherwise }\end{cases} \\
& \quad l_{i} \in I_{m}-\{m\}
\end{aligned}
$$

Proof: Straightfoward results from the definition of SSMFs.

It is not necessary to calculate the membership values for all SSMFs owing to this property. This greatly reduces the computational effort in implementing SSMFs in industrial processes.

Property 2: The two nonzero membership values in the SSMFs of property 1 have a sum of 1 and hence the summation of all membership values for $x_{i}$ is also 1 . That is

$$
\sum_{l_{i} \in I_{m}} A_{\left(i, l_{i}\right)}\left(x_{i}^{*}\right)=1 \quad \forall x_{i}^{*} \in U_{x_{i}}
$$

Proof: Straightforward results from the definition of SSMFs.

The following property states that the centroid of a single membership function (local centroid) for output linguistic variable with the form of eqn. 19 can be computed by using the principal values only.

Property 3: For the SSMFs $B_{l}(y)$ s defined in eqn. 19 , let $c_{l} \mathrm{~s}$ be the centroids of membership functions $B_{l}(y) \mathrm{s}$, then

$$
c_{l}=\frac{1}{3}\left(B_{l-1}^{*}+B_{l}^{*}+B_{l+1}^{*}\right) \quad \text { for } l \in I_{m_{y}}
$$

Proof: In computing the centroid value for membership function $B_{l}(y)$, the widely used centre of area (COA) method is adopted here to generates the result. In the case of a continuous universe of discourse, this method gives

$$
c_{l}=\frac{\int_{U_{y}} B_{l}(y) y d y}{\int_{U_{y}} B_{l}(y) d y}
$$

First, for $l \in I_{m y}-\left\{-m_{y}, m_{y}\right\}$ let $B_{l}=\Lambda\left(y ; B_{l-1}, B_{l}^{*}, B_{l+1}^{*}\right)$, and the numerator of eqn. 22 is referred to as the moment and the denominator is called the area of the membership function for convenience. Then the moment $M_{l}$ of the membership function $B_{l}$ is

$$
\begin{aligned}
M_{l} & =\int_{U_{y}} B_{l}(y) y d y \\
& =\int_{B_{l-1}^{*}}^{B_{l}^{*}}\left(\frac{y-B_{l-1}^{*}}{B_{l}^{*}-B_{l-1}^{*}}\right) y d y+\int_{B_{l}^{*}}^{B_{l+1}^{*}}\left(\frac{B_{l+1}^{*}-y}{B_{l+1}^{*}-B_{l}^{*}}\right) y d y \\
& =\frac{1}{6}\left(B_{l+1}^{*}-B_{l-1}^{*}\right)\left(B_{l-1}^{*}+B_{l}^{*}+B_{l+1}^{*}\right)
\end{aligned}
$$


The area $S_{l}$ is

$$
\begin{aligned}
S_{l} & =\int_{U_{y}} B_{l}(y) d y \\
& =\int_{B_{l-1}^{*}}^{B_{l}^{*}}\left(\frac{y-B_{l-1}^{*}}{B_{l}^{*}-B_{l-1}^{*}}\right) d y+\int_{B_{l}^{*}}^{B_{l+1}^{*}}\left(\frac{B_{l+1}^{*}-y}{B_{l+1}^{*}-B_{l}^{*}}\right) d y \\
& =\frac{1}{2}\left(B_{l+1}^{*}-B_{l-1}^{*}\right)
\end{aligned}
$$

Therefore the centroid of membership function $B_{l}$ is

$$
c_{l}=\frac{M_{l}}{S_{l}}=\frac{1}{3}\left(B_{l-1}^{*}+B_{l}^{*}+B_{l+1}^{*}\right)
$$

As for $l \in\left\{m_{v},-m_{y}\right\}$, the same result can be obtained by restricting the integration range within the universe of discourse. This completes the proof.

The following theorem states that the final crisp output of the SSMFs-FLC can be computed from the local centroids with a discrete method. This result lessens computation and favour digital implementation.

Theorem 1: For a SSMFs-FLC with its membership functions described in eqns. 18 and 19, suppose the algebraic product is chosen as operator $\mathbf{F}$ and $\mathbf{I}$, summation $(\Sigma)$ is chosen as operator $\mathbf{A}$, and COA is used for defuzzification. Given the crisp input values $x_{i}^{*} \mathrm{~s}$, then the final crisp output $y^{*}$ can be computed from local centroids:

$$
y^{*}=\frac{\sum_{l=-m_{y}}^{m_{y}} c_{l} S_{l} \Phi_{l}}{\sum_{l=-m_{y}}^{m_{y}} S_{l} \Phi_{l}}
$$

here $c_{l}$ and $S_{l}$ are the centroid and area of the $l$ th membership function of output linguistic variable $y, \Phi_{l}$ is the summation of the firing levels of all rules whose consequent fuzzy sets equal the th fuzzy set of $y$, that is

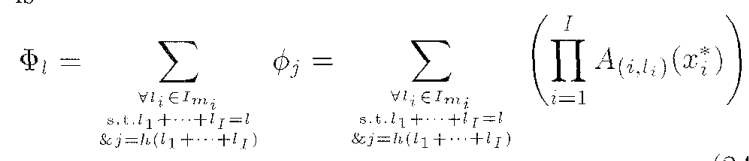

where $A\left(i, l_{i}\right)\left(x_{i}^{*}\right)$ s are the membership values of inputs $x_{i}^{*} \mathrm{~s}$.

Proof: According to the inference procedures proposed for SSMFs-FLC, we first use the sequence representation of the rule base to show that the crisp output of the system described in theorem 1 can be obtained from local centroids and firing levels, and then perform a transformation to the index representation to get the final results.

Starting from the primitive eqn. 9 and applying the proper operators, we get

$$
\begin{aligned}
y^{*}= & \frac{\int_{U_{y}} y B(y) d y}{\int_{U_{y}} B(y) d y}=\frac{\int_{U_{y}} y\left(\sum_{j=1}^{J} \phi_{j} B_{j}(y)\right) d y}{\int_{U_{y}}\left(\sum_{j=1}^{J} \phi_{j} B_{j}(y)\right) d y} \\
= & \frac{\sum_{j=1}^{J}\left(\phi_{j} \int_{U_{y}} y B_{j}(y) d y\right)}{\sum_{j=1}^{J}\left(\phi_{j} \int_{U_{y}} B_{j}(y) d y\right)}
\end{aligned}
$$

here, $\phi_{j}$ is the firing level of the $j$ th rule and equals $\Pi_{i=1}^{I} A_{(i, j)}\left(x_{i}^{*}\right)$. Let $S_{j}$ and $c_{j}$ be the respective area and centroid of the $j$ th rule's consequent set $B_{j}$ :

$$
\begin{aligned}
& S_{j}=\int_{U_{y}} B_{j}(y) d y \\
& c_{j}=\frac{\int_{U_{y}} y B_{j}(y) d y}{\int_{U_{y}} B_{j}(y) d y}=\frac{\int_{U_{y}} y B_{j}(y) d y}{S_{j}}
\end{aligned}
$$

substituting from eqn. 26. Hence

$$
\int_{L_{y}} y B_{j}(y) d y=c_{j} S_{j}
$$

Substituting eqns. 26 and 27 into eqn. 25 , we derive

$$
y^{*}=\frac{\sum_{j=1}^{J} c_{j} S_{j} \phi_{j}}{\sum_{j=1}^{J} S_{j} \phi_{j}}
$$

As noted previously, the sequence representation of rule base can be mapped to index representation, eqns. 14 and 13 serving the purpose of transformation between these two representations. From the relations given there may be several js mapping to the same $l$, that is there are several rules having the identical consequent set. Substituting $l$ for $j$ in eqn. 28 and sum the firing levels of the same consequent set into a single term, we get the final form for the centroid:

$$
y^{*}=\frac{\sum_{l=-m_{y}}^{m_{y}} c_{l} S_{l} \Phi_{l}}{\sum_{l=-m_{y}}^{m_{y}} S_{l} \Phi_{l}}
$$

where $\Phi_{l}$ is the summation of the firing levels of all rules whose consequent fuzzy sets equals the $l$ th fuzzy set of $y$, as defined in eqn. 24 .

In summary, the SSMFs-FLC is more rational than the FLC in both theory and implementation. Due to the features of fuzzy sets, SSMFs-FLC can deal with the indistinctness of some processes that we cannot describe with real precision. Moreover, the SSMFs provide SSMFs-FLC another equipment to handle the obstacles in constructing membership functions for an FLC. By only setting $m$ and $s$, a series of membership functions with reasonable physical meaning are attained for complicated processes. Further, with proper parameters of SSMFs the calculation of the controller is lessened dramatically. All these make the SSMFs-FLC a promising implementation over the conventional FLC.

In the following Section we apply the SSMFs-FLC to some representative processes to demonstrate its utility.

\section{Simplified SSMFs-FLC and its performance}

In this Section, some numerical simulations are used to examine both the effects of the design parameters and the ability of SSMFs-FLC. First, the effects of shrinking factor are illustrated by observing the changes occurred in the control surfaces when different shrinking factors are applied to the two-input one-output system. Next, some typical processes are used to examine the control ability of SSMFs-FLC on linear systems which have same designing parameters $(m$ and $s$ ) of 
SSMFs-FLC. Finally, the SSMFs-FLC is applied to a pendulum-car system to demonstrate both the effects of shrinking factors on control performances and the robustness of the SSMFs-FLC on different initial conditions.

\subsection{Simplified SSMFs-FLC}

Although the SSMFs-FLC is designed as a multi-input one-output FLC, the two-input one-output system is adopted here as an example. We refer this type of SSMFs-FLC as a simplified SSMFs-FLC.

For an ordinary feedback control scheme, the inputs of the SSMFs-FLC used in this Section are error $e$ and change in error $r$ of the process, the output linguistic variable is the change in controller output $u$. The associative universe of discourse and term-sets of these linguistic variables are $U_{e}$ and $T_{e}, U_{r}$ and $T_{r}$, and $U_{u}$ and $T_{u}$ for $e, r$ and $u$, respectively.

In this study, simple rule mapping is applied to construct the rule base and the inference procedures described in eqn. 9 are used to calculate the result for the following cases. Some assumptions are made beforehand: $U_{e}, U_{r}$ and $U_{u}$ are all set to $[-1,1]$; the term-sets $T_{e}$ and $T_{r}$ have the same number of elements, that is $m_{e}=m_{r}=m$ and therefore $m_{u}=m_{e}+m_{r}=2 m$. The subscripts $i, j$ and $k(=i+j)$ are associated with $e, r$ and $u$, respectively, as indices. The typical two-input one-output SSMFs-FLC feedback control system and its detailed computational steps are depicted in Figs. 3 and 4.

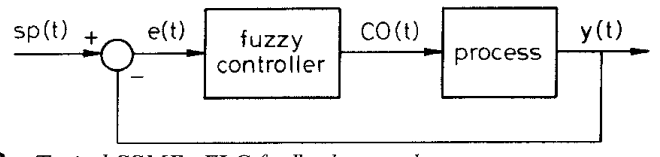

Fig 3 Typical SSMFs-FLC feedback control system

The notations therein are defined as follows:

$$
\begin{aligned}
e_{m}(n) & =\left.\{s p(t)-y(t)\}\right|_{t=n T} \\
r_{m}(n) & =\frac{e_{m}(n)-e_{m}(n-1)}{T} \\
e^{*} & =\operatorname{GE} e_{m}(n) \\
r^{*} & =\operatorname{GR} r_{m}(n) \\
E_{i}\left(e^{*}\right) & =\mathbf{M}\left(e^{*}, E_{i}\right) \\
R_{i}\left(r^{*}\right) & =\mathbf{M}\left(r^{*}, R_{i}\right) \\
U(u) & =\underset{\forall i, j \in I_{m}}{\mathbf{A}}\left(\mathbf{I}\left(\mathbf{F}\left(E_{i}\left(e^{*}\right), R_{j}\left(r^{*}\right)\right), U_{k=i+j}(u)\right)\right) \\
u^{*} & =\mathbf{D}(U(u)) \\
u_{m}(n) & =\mathrm{GU} u^{*} \\
\mathrm{CO}(n) & =\mathrm{CO}(n-1)+u_{m}(n)
\end{aligned}
$$

here, $T$ is the sampling interval; $s p(t)$ is the set point $\mathrm{CO}(t)$ is the controller output and $y(t)$ is the process output; $e_{m}(n), r_{m}(n)$ and $u_{m}(n)$ are error, rate of change in error and change in controller output, respectively, at the $n$th sampling point. GE, GR and GU are scalers for $e_{m}(n), r_{m}(n)$, and $u_{m}(n)$, respectively, and the scaled crisp value $e^{*}, r^{*}$ and $u^{*}$ have a numerical range in $[1,-1] . I_{m e}, I_{m r}$ and $I_{m u}$ are the index sets for the termsets of linguistic variable $e, r$ and $u$, respectively; $E_{i}\left(e^{*}\right)$ and $R_{i}\left(r^{*}\right)$ are the membership grade for $e$ and $r$, respectively; $U_{k}(u)$ are membership functions for $u$. Operators $\mathbf{M}, \mathbf{F}, \mathbf{I}, \mathbf{A}$ and $\mathbf{D}$ are defined in equs. 2, 4, 5, 6 and 7 , respectively.

\subsection{Control surface of simplified SSMFs-FLC}

The effects of shrinking factors on FLC can be examined by comparing the control surface of different sets of shrinking factors. Here we present four control surfaces (Figs. 5-8) with various values of shrinking factor. In the implementation the algebraic product is chosen as operators $\mathbf{F}$ and $\mathbf{I}$, summation $(\Sigma ;)$ is picked as operator A, COA is used for defuzzification operator $\mathbf{D}$. One can find that the control surfaces are quite different for distinct values of shrinking factors. Therefore by modifying shrinking factors the human operator can acquire numerous control surfaces and then pick up the one that is most suitable.

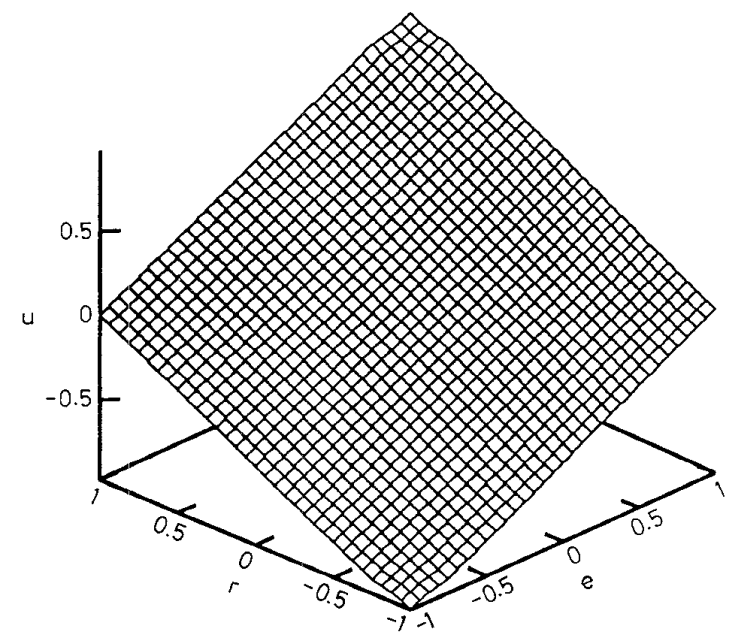

Fig.5 Typical control surfaces of simplified SSMFS-FLC $s_{e}=s_{r}=s_{u}=$ 10

From Figs. 5-8 one can find that the control surface of the SSMFs-FLC with equal-span membership functions ( $s_{e}=s_{r}=s_{u}=1$ ) is a linear one, and thus is obviously not adequate to control the nonlinear processes. On the other hand, an FLC with nonlinear control

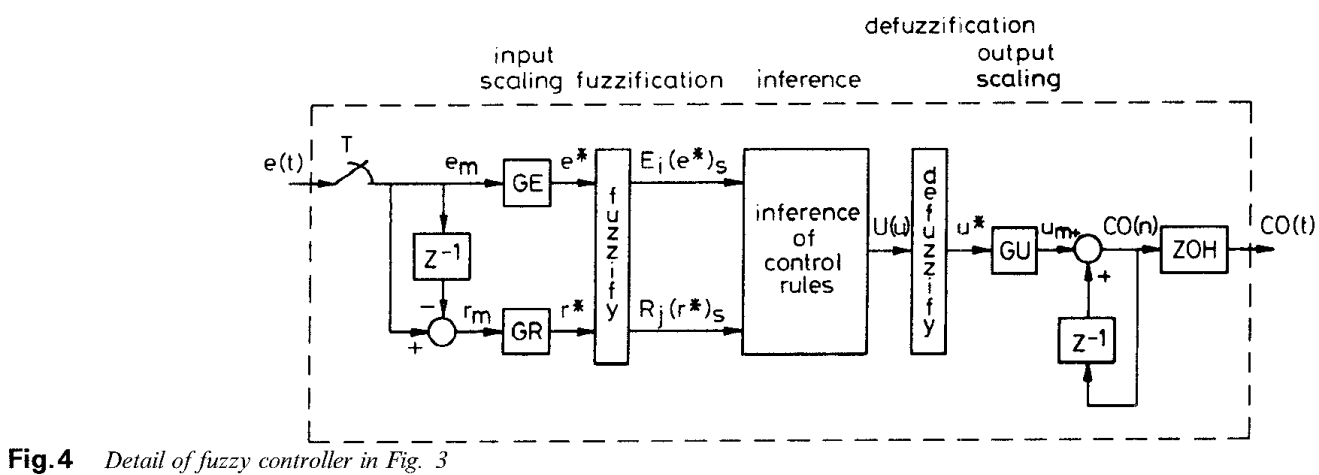




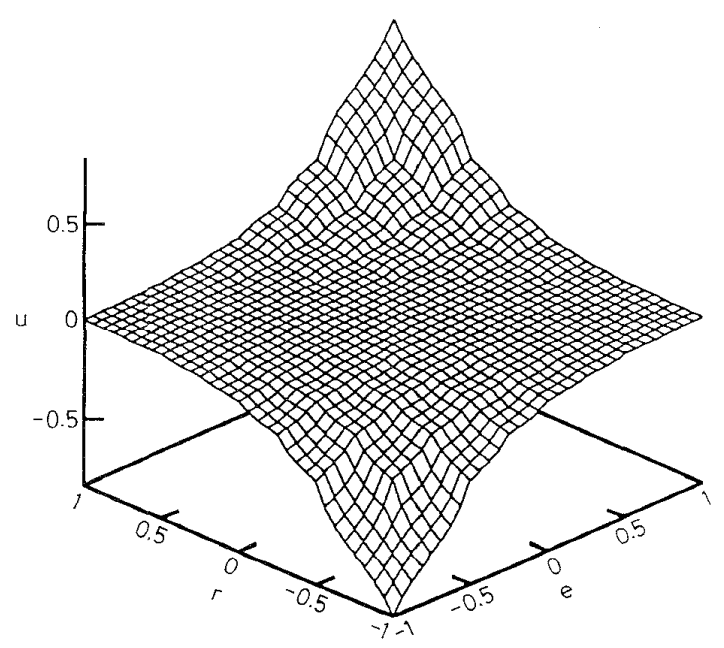

Fig.6 Typical control surfaces of simplified SSMFs-FLC $s_{e}=s_{r}=0.6$

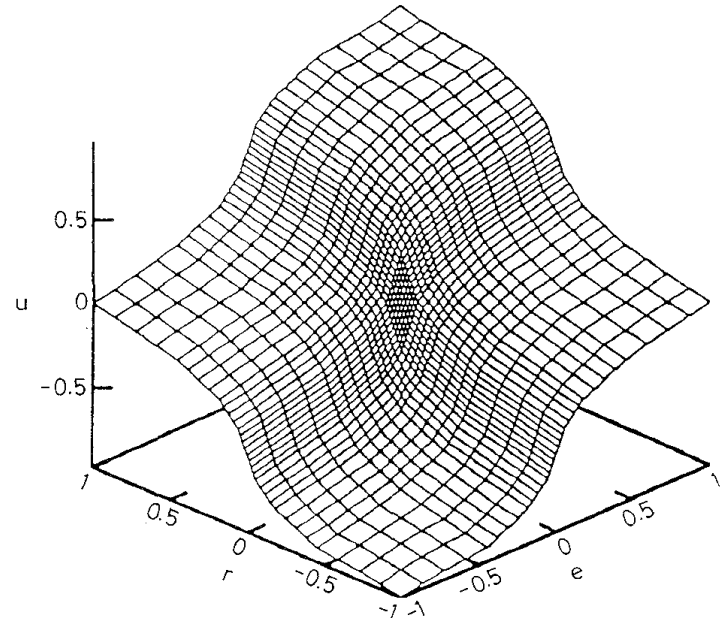

Fig.7 Typical control surfaces of simplified SSMFs-FLC $s_{e}=s_{r}=1.0$; $s_{u}=0.6$

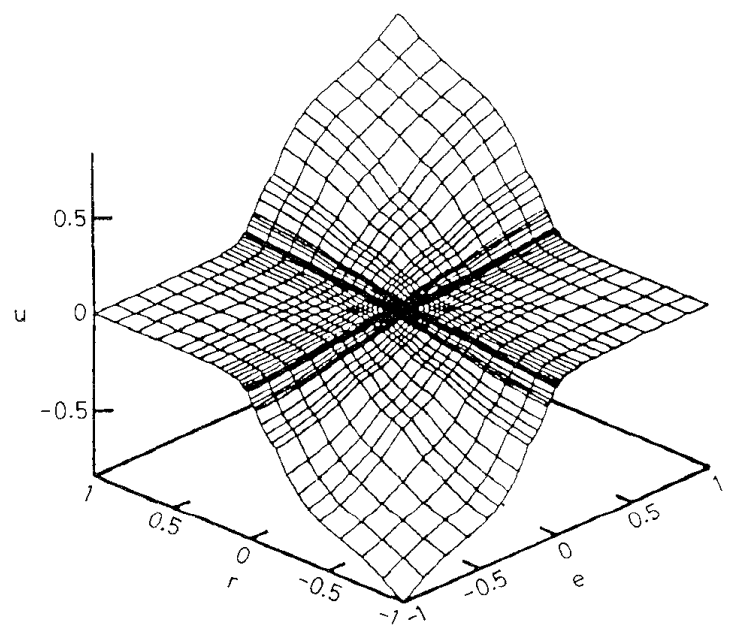

Fig.8 Typical control surfaces of simplified SSMFs-FLC $s_{e}=s_{r}=s_{u}=$

surface may not be a good one to handle the linear processes. For that reason, the characteristics of the control surface can be modified by only the shrinking factors is clearly a merit of the SSMFs-FLC.

\subsection{Control response of SSMFs-FLC on linear} processes

Five linear processes are used to explore the feasible nominal performance of the SSMFs-FLC:

(i) TY: typical first-order with dead-time process

$$
G_{p}(s)=\frac{e^{-0.2 s}}{s+1}
$$

(ii) LD: first-order with large dead-time process

$$
G_{p}(s)=\frac{e^{-4 s}}{s+1}
$$

(iii) UD: underdamped process

$$
G_{p}(s)=\frac{e^{-s}}{9 s^{2}+2.4 s+1}
$$

(iv) $\mathrm{HO}$ : high-order process

$$
G_{p}(s)=\frac{e^{-3 s}}{(s+1)^{2}(s 2+1)}
$$

(v) IR: inverse response process

$$
G_{p}(s)=\frac{(-s+1) e^{-s}}{(2 s+1)(s+1)}
$$

The same number of linguistic members and shrinking factors are assigned to all the five cases to show the generality of the SSMFs-FLC. The only tuning parameters are input/output scalers (GE, GR, GU) which are tuned by the rules-of-thumb. Two principles must be observed in tuning the parameters:

(a) GE and GR must appropriately transfer the sensor data, error and change in error, into the specific universe of discourse, say $[-1,1]$.

(b) The control action should not go beyond the physical capability of the controller, that is, GU is confined to a certain range.

The number of linguistic members is set to nine for both error and rate of change in error, that is $m_{e}=m_{r}$ $=4$, thus the number of linguistic members for change in controller output is therefore $17\left(m_{u}=m_{e}+m_{r}\right)$.

Table 2 shows the design parameters $\left(m_{e}, m_{r}, s_{e}, s_{r}\right.$, $s_{u}$ ), tuning parameters (GE, GR, GU) used and resulting IAE of the five demonstrated cases for $10 \%$ set point response. Fig. 9 shows the set-point responses, i.e. set point is changed from 50 to $60 \%$.
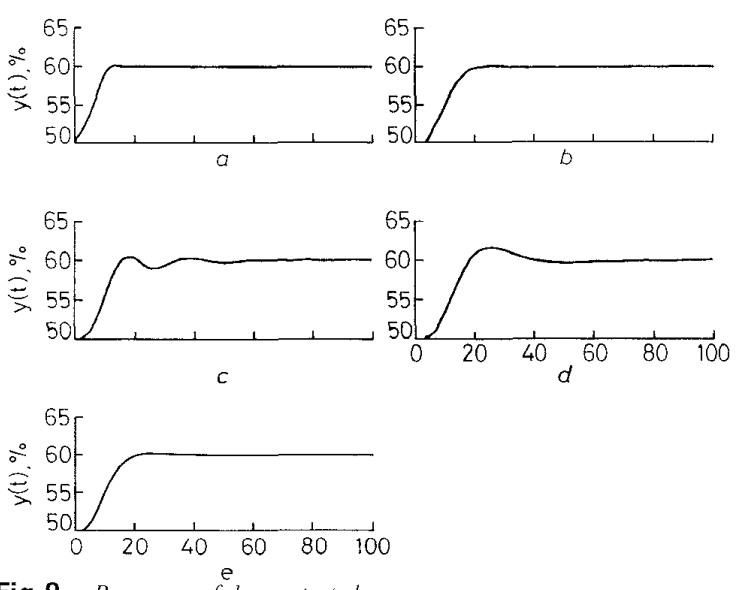

Fig.9 Responses of demonstrated cases

a Typical first-order process

$b$ Large dead time process

c Underdamped process

d High-order process

e. Inverse response process

IEE Proc-Control Theory Appl., Vol. 143, No, 4, July 1996 
Note that the shrinking factors are arbitrarily selected in these examples. Since optimisation is not involved in the procedures for obtaining the values of GE, GR, GU, the performances should be viewed with an eye to their rationality rather than their superiority.

Table 2: Design and tuning parameters of demonstrated cases

\begin{tabular}{|c|c|c|c|c|c|c|c|}
\hline \multirow[t]{2}{*}{ Case } & \multicolumn{3}{|c|}{ Shrinking factors } & \multirow[t]{2}{*}{ GE } & \multirow[t]{2}{*}{ GR } & \multirow[t]{2}{*}{ GU } & \multirow[t]{2}{*}{ IAE } \\
\hline & $S_{e}$ & $S_{r}$ & $S_{u}$ & & & & \\
\hline$T Y$ & & & & 0.150 & 0.03 & 3.00 & 61.21 \\
\hline LD & & & & 0.050 & 0.01 & 1.50 & 106.89 \\
\hline UD & 0.90 & 0.90 & 0.75 & 0.075 & 0.02 & 1.00 & 108.87 \\
\hline $\mathrm{HO}$ & & & & 0.075 & 0.02 & 1.00 & 142.49 \\
\hline IR & & & & 0.075 & 0.02 & 1.00 & 106.28 \\
\hline
\end{tabular}

\subsection{Control response of SSMFs-FLC on nonlinear process}

From the control surface of the SSMFs-FLC, one can find that the SSMFs-FLC possesses a nonlinear feature in its control structure. To exhibit its control ability for nonlinear processes, the SSMFs-FLC is applied to a pendulum-car system to achieve this aim.

The pendulum-car system $[15,16]$ consists of:

- a car moving along a line on two rails of limited length

- a pendulum hinged in the car by means of ball bearings rotating freely in the plane containing this line

- a car driving device containing a DC motor, a DC amplifier, and a pulley-belt transmission system

Such a system is characterised by an unstable equilibrium point in the upright position of the pendulum, a stable equilibrium point in pendant position, as well as two uncontrollable points when the pendulum is horizontal. The dynamic behaviour of the pendulum-car system can be derived $[15,16]$ and expressed as

$\theta^{\prime \prime}=\frac{\left[\left(M_{c}+M_{p}\right) g \tan \theta+\frac{1}{2} M_{p} L \sin \theta \cdot \theta^{\prime 2}\right]+u}{-\frac{2}{3}\left(M_{c}+M_{p}\right) L \sec \theta+\frac{1}{2} M_{p} L \cos \theta}$

where the $M_{p}$ and $l$ are the mass and length of the pendulum, respectively, $M_{c}$ is the mass of the car, $\theta$ is the angle measured from the pendant position, and $u$ is the driving force applied to the car. [16]:

The parameters of the model are taken as follows

$M_{c}=2.8 \mathrm{~kg} ; M_{p}=0.2 \mathrm{~kg} ; L=0.75 \mathrm{~m} ; g=9.81 \mathrm{~m} / \mathrm{s}^{2}$

The control objective is to stabilise the pendulum in upright $\left(180^{\circ}\right)$ position from an initial deflect position (say $170^{\circ}$ ) by changing the driving force $u$. Due to the characteristics of the pendulum-car system, the control scheme is slightly modified so that the output linguistic variable is the controller output, rather than the change in controller output. It means that the controller output $\mathrm{CO}(t)$ no longer equals $\mathrm{CO}(t-1)+u_{m}(n)$ but equals $u_{m}(n)$ directly.

The main aim of the simulation is to demonstrate the effects of both the shrinking factors and robustness of the SSMFs-FLC for nonlinear systems.

4.4.1 Effects of shrinking factors: The effects of shrinking factors on control performance are demon- strated by applying different combinations of $s_{e}, s_{r}, s_{u}$ to the SSMFs-FLC with constant parameters $(m=4$, $\mathrm{GE}=0.5, \mathrm{GR}=0.01, \mathrm{GU}=300$ ), the initial position of the pendulum is $170^{\circ}$, the initial control value is 0 , and the sampling interval in this simulation is set to $10 \mathrm{~ms}$. To make the differences between the responses clearer, $s_{v}$ and $s_{r}$ are set to $0.9,0.6$ and $0.3 ; s_{u}$ is changed from 1.0 to 0.7 to 0.4 . Fig. 10 shows the responses of the nine combinations of shrinking factors. One can see that the responses differ markedly between these simulations. Some trends can be observed from the responses: for the pendulum-car system: the less are $s_{e}$ and $s_{r}$, the less the overshoot and oscillation; the less is $s_{u}$, the slower the response. This is due to the fact that modifying the shrinking factors not only alters the layout of the membership functions of the linguistic variables but also changes the rules fired for the identical inputs. Certainly, the trends mentioned may not occur for other nonlinear systems, however, the effects of shrinking factors are already elucidated by this example.
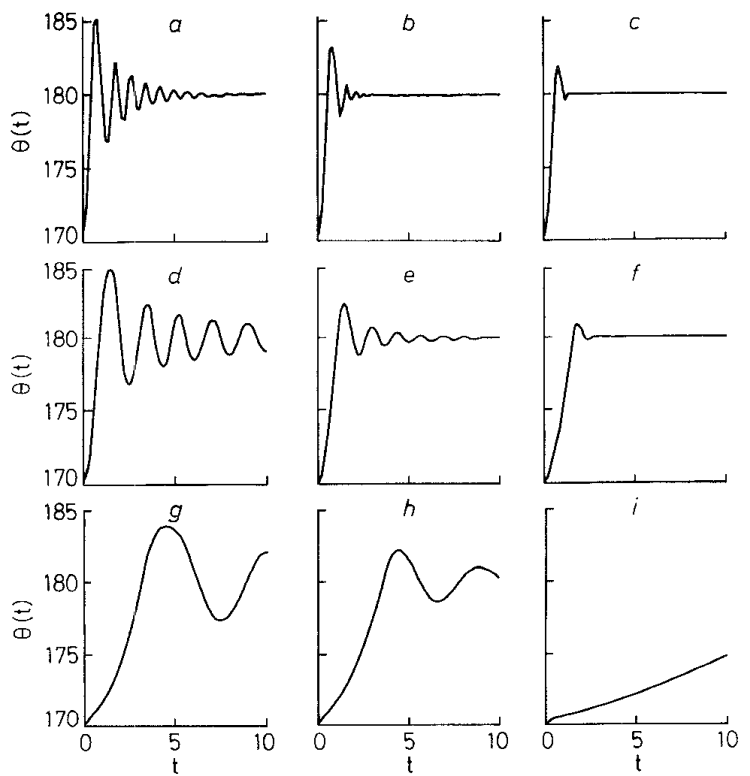

Fig. 10 Response of $\theta(t)$ using different shrinking factors

$\left(\theta(0)=170^{\circ}\right.$

(a) $s_{e}=s_{r}=0.9 ; s_{u}=1.0$

(b) $s_{e}=s_{r}=0.6 ; s_{u}=1.0$

(c) $s_{e}=s_{r}=0.3 ; s_{u}=1.0$

(d) $s_{u^{\prime}}=s_{r}=0.9 ; s_{u}=0.7$

(e) $s_{c}=s_{r}=0.6 ; s_{u}=0.7$

(f) $s_{e}=s_{r}=0.3 ; s_{u}=0.7$

(g) $s_{e}=s_{r}=0.9, s_{u}=0.4$

(h) $s_{u}=s_{r}=0.6 ; s_{u}=0.4$

(i) $s_{i}=s_{r}=0.3 ; s_{u}=0.4$

4.4.2 Robustness of SSMFS-FLC: The robustness of SSMFs-FLC is showed by changing the initial position of the pendulum. In this example, the shrinking factors of the SSMFs-FLC are $s_{e}=s_{r}=0.3$ and $s_{u}=$ 1.0 , the other parameters are the same as those in Fig. 10 . These values are originally used to control the pendulum with the initial position of $170^{\circ}$, however, the same values are applied to control the other three pendulums with initial position of 150,130 , and $110^{\circ}$, respectively, to examine the robustness of the SSMFsFLC. The simulation results are plotted in Fig. 11. All the four pendulums reach the set point with little oscillation and then maintain their positions at $180^{\circ}$ perfectly. 

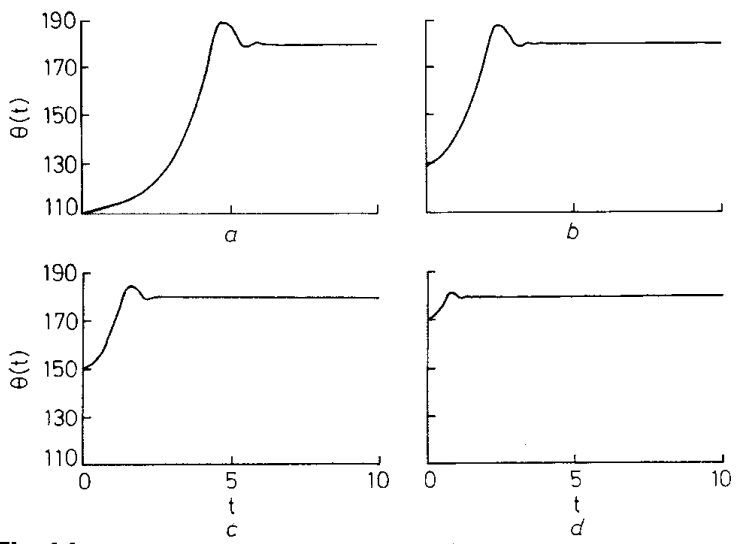

Fig. 11 Response of $\theta(t)$ starting with different initial positions $s_{E}=S_{R}=0.3 ; s_{U}=1.0$

$a \theta(0)=110^{\circ}$

$b \theta(0)=130^{\circ}$

c $\theta(0)=150^{\circ}$

$d \theta(0)=170^{\circ}$

From these empirical studies, the desired control actions of the SSMFs-FLC can be obtained readily by carefully choosing the appropriate shrinking factors. Moreover, the SSMFs-FLC has shown its ability as an efficient and yet robust control scheme for either linear and nonlinear processes.

\section{Conclusion}

We have proposed a new scheme, shrinking-span membership functions, to specify the membership functions for a fuzzy logic controller. With only two parameters (shrinking factor and number of linguistic variables), this method provides an easy and systematic way in designating membership functions and the membership functions generated are more reasonable to the human perception than those with equal spans. When lacking thorough knowledge of the process, the human operator can use both SSMFs and simple rule mapping to construct a primitive FLC which can be used as a fundamental structure for further fine tuning.

According to the simulation results, the SSMFs-FLC shows its control ability in either linear and nonlinear systems. Satisfactory responses can be acquired without laborious optimisation calculation. Further, the SSMFs-FLC also exhibits its robustness in controlling the highly nonlinear pendulum-car system with very different initial conditions. All these features demonstrate that the SSMFs-FLC possesses the capability to adapt itself to different system deviations easily.

Since it reduces both the domain knowledge of the process and the load of computation in devising an FLC, the SSMFs-FLC narrows the gap between a theoretical FLC and a practical one, and thus helps the FLC more realistic.

\section{References}

1 ZADEH, L.A.: 'Fuzzy sets', Inform. Control, 8, pp. 338-353

2 MAMDANI, E.H.: 'Applications of fuzzy algorithms for simple dynamic plant', IEE Proc., 1974, 121, pp. 1585-1588

3 MAMDANI, E.H., and ASSILIAN, S.: 'An experiment in linguistic synthesis with a fuzzy logic controller', Int. J. Man-Mach. Stud, 1975, 7, pp. 1-13

4 LEE, C.C.: Fuzzy logic in control systems: Fuzzy logic controller, Part I, II', IEEE Trans., 1990, SMC-20, pp. 404435

5 RAIU, G., and ZHOU, J.: 'Adaptive hierarchical fuzzy controller', IEEE Trans., 1993, SMC-23, pp. 973-980

6 WONG, C., CHOU, C., and MON, D.: 'Studies on the output of fuzzy controller with multiple inputs', Fuzzy Sets Syst., 1993, 57, pp. $149-158$

7 TANG, K., and MULHOLLAND, R.: 'Comparing fuzzy logic with classical controller design', IEEE Trans., 1987, SMC-17, pp. $1085-1087$

8 SILER, W., and YING, H.: 'Fuzzy control theory: The linear case', Fuzzy Sets Syst., 1989, 33, pp. 275-290

9 YING, H., SILER, W., and BUCKLEY, J.J.: 'Fuzzy control theory: A nonlinear case', Automatica, 1990, 26, pp. 513-520

10 CHEN, C.-L., and CHEN, W.-C.: 'Fuzzy controller design by using neural network techniques', IEEE Fuzzy Syst., 1994, 2, pp. 235-244

11 DRIANKOV, D., HELLENDOOM, H., and REINFRANK, M: 'An introduction to fuzzy control' (Springer-Verlag, Berlin, 1993)

12 YAGER, R.R., and FILEV, D.P. 'Essentials of fuzzy modeling and control' (Wiley, New York, 1994)

13 HELLENDOORN, H.: 'Closure properties of the compositional rule of inference', Fuzzy Sets Syst., 1990, 35, pp. 163-183

14 MIZUMOTO, M.: 'Pictorial representations of fuzzy connectives, Part I: cases of t-norms, t-conorms and averaging operators', Fuzzy Sets Syst., 1989, 31, pp. 217-242

15 CZOGALA, E., MROZEK, A., and PAWLAK, Z.: "The idea of a rough fuzzy controller and its application to the stabilisation of a pendulum-car system', Fuzzy Sets Syst., 1995, 72, pp. 61-73

16 LIN, E.C., and SHEU, Y.-R.: 'A hybrid-control approach for pendulum-car control', IEEE Trans., 1992, IE-39, pp. 208-214 